\title{
Cooperative control of integrator negative imaginary systems with application to rendezvous multiple mobile robots
}

DOI:

10.1109/RoMoCo.2019.8787358

\section{Document Version}

Accepted author manuscript

Link to publication record in Manchester Research Explorer

Citation for published version (APA):

Skeik, O., Hu, J., Arvin, F., \& Lanzon, A. (2019). Cooperative control of integrator negative imaginary systems with application to rendezvous multiple mobile robots. In Proceedings of the 12th International Workshop on Robot Motion and Control https://doi.org/10.1109/RoMoCo.2019.8787358

\section{Published in:}

Proceedings of the 12th International Workshop on Robot Motion and Control

\section{Citing this paper}

Please note that where the full-text provided on Manchester Research Explorer is the Author Accepted Manuscript or Proof version this may differ from the final Published version. If citing, it is advised that you check and use the publisher's definitive version.

\section{General rights}

Copyright and moral rights for the publications made accessible in the Research Explorer are retained by the authors and/or other copyright owners and it is a condition of accessing publications that users recognise and abide by the legal requirements associated with these rights.

\section{Takedown policy}

If you believe that this document breaches copyright please refer to the University of Manchester's Takedown Procedures [http://man.ac.uk/04Y6Bo] or contact uml.scholarlycommunications@manchester.ac.uk providing relevant details, so we can investigate your claim.

\section{OPEN ACCESS}




\title{
Cooperative control of integrator negative imaginary systems with application to rendezvous multiple mobile robots
}

\author{
Ola Skeik, Junyan Hu, Farshad Arvin, and Alexander Lanzon
}

\begin{abstract}
This paper contributes to the solution of mobile robots rendezvous problem via the negative imaginary (NI) systems theory. Cooperative control strategies are proposed for integrator NI systems. These control strategies are beneficial because they can be directly applied to wheeled mobile robots. First, we show that the NI property is preserved for multiple multi-input multi-output (MIMO) integrator systems with directional information flow that is balanced and strongly connected. Then, we derive conditions that guarantee output consensus and output tracking for strongly connected, balanced and directed networks of integrators subject to energy-bounded disturbances using the NI internal stability theorems. Finally, experimental and simulation results are provided to validate the effectiveness of the proposed NI cooperative tracking results to achieve rendezvous of multiple nonholonomic wheeled mobile robots.
\end{abstract}

\section{INTRODUCTION}

Multiple mobile robot cooperation is an important field of study as mobile robots have various applications that benefit the society such as cooperative rescue missions in hazardous environments. The area has been widely explored by researchers over the past decade such as [1]-[3] to name a few. In multiple mobile robot cooperation, robots cooperate with each other in order to achieve a desired collective behaviour (or accomplish a complex task) via distributed control laws. Common collective behaviours include consensus, tracking a reference, rendezvous, synchronization, etc. However, control of multiple mobile robots is challenging due to limitations in the shared information and uncertainties in the robots environment. Consequently, for a group of robots to successfully accomplish a complex task (or desired collective behaviour), advanced control strategies and distributed control laws need to be developed. In this paper, we choose the negative imaginary (NI) systems theory to tackle the cooperative control problem (specifically rendezvous) for multiple nonholonomic wheeled mobile robots (WMR). The reason for this choice is because the dynamics of nonholonomic WMR can be simplified to a single integrator model via input-output linearisation making it possible to develop simple yet sophisticated control laws for integrator NI systems in a systematic way which can be directly applied to WMR.

This work was supported by the Engineering and Physical Sciences Research Council (EPSRC) [grant number EP/R008876/1] and a University of Manchester President's Doctoral Scholar Award. All research data supporting this publication are directly available within this publication.

The authors are with the Control Systems Centre, School of Electrical and Electronic Engineering, University of Manchester, Sackville St., Manchester, M13 9PL, UK. (e-mail: ola.skeik@manchester.ac.uk, junyan.hu@manchester.ac.uk, alexander.lanzon@manchester.ac.uk).
Negative imaginary systems theory was first introduced in [4] for the purpose of tackling robust stability issues related to lightly damped flexible structures with force inputs and position outputs. Recent theoretical studies and applications regarding NI systems can be found in [5]-[7] to name a few. The NI systems theory has been recently applied to solve multi-agent systems problems in [8]-[11]. Particularly, robust output consensus was addressed in [8] and [9] for networks of homogeneous and heterogeneous NI systems respectively by reformulating the consensus problem into an internal stability problem. Moreover, robust output tracking for multiple NI systems was addressed in [9]. However, the works in [8], [9] only dealt with undirected graphs to model the interaction among the systems. Whilst the results in [8], [9] are not applicable to directed graphs, we proof here that for multi-input multi-output (MIMO) integrator NI systems and for directed graphs that are balanced and strongly connected the consensus and tracking problems can be guaranteed via the NI internal stability theorems. We then make a practical and effective use of these results to solve a rendezvous problem for multiple WMR.

In this paper, we first propose the main results for integrator NI systems, then we utilize the results to achieve rendezvous of multiple WMR. We build on the works of [8] and [9]. We proof that multiple integrator NI systems with directional information flow that is balanced and strongly connected retains the NI property. Subsequently, we derive conditions, using NI systems theory, such that output consensus and cooperative tracking are guaranteed for a network of integrators with strongly connected, balanced and directed information flow subject to energy-bounded disturbances. Experimental results from both real-robot and simulation are provided to validate the effectiveness of the proposed theoretical results in solving a rendezvous problem for multiple WMR.

Notation: Let $\mathbb{R}^{m \times n}$ denote the set of $m \times n$ real matrices. Given a matrix $A, A^{T}$ and $A^{*}$ are the transpose and the complex conjugate transpose of $A$ respectively. $\Re[\cdot]$ is the real part of a complex number. $I_{N}$ is the identity matrix of dimension $N \times N$ and $1_{N}$ is an $N \times 1$ vector with entries 1. $A \otimes B$ denotes the Kronecker product of matrices $A$ and $B . \mathbb{N}(A)$ is the null space of matrix $A .[P, K]$ represents a positive feedback interconnection between systems $P(s)$ and $K(s)$.

\section{Preliminaries}

\section{A. Negative Imaginary Systems}

A negative imaginary system is defined as follows. 
Definition 1 ([12]): A square, real, rational, proper transfer function matrix $P(s)$ is said to be negative imaginary if

1) $P(s)$ has no poles in $\Re[s]>0$;

2) For all $\omega>0$ such that $j \omega$ is not a pole of $P(s)$, $j\left(P(j \omega)-P(j \omega)^{*}\right) \geq 0$

3) If $s=j \omega_{0}$ with $\omega_{0}>0$ is a pole of $P(s)$, then it is a simple pole and the residue matrix $K=\lim _{s \rightarrow j \omega_{0}}(s-$ $\left.j \omega_{0}\right) j P(s)$ is Hermitian and positive semi-definite;

4) If $s=0$ is a pole of $P(s)$, then $\lim _{s \rightarrow 0} s^{r} P(s)=0$ for all $r \geq 3$ and $\lim _{s \rightarrow 0} s^{2} P(s)$ is Hermitian and positive semi-definite.

A strictly negative imaginary (SNI) system is defined as follows.

Definition 2 ([4], [13]): A square, real, rational, proper transfer function matrix $K(s)$ is said to be strictly negative imaginary if

1) $K(s)$ has no poles in $\Re[s] \geq 0$;

2) $j\left[K(j \omega)-K(j \omega)^{*}\right]>0$ for all $\omega \in(0, \infty)$.

The follow lemma provides an internal stability result when the NI system has a single pole at the origin.

Lemma 1 ([12]): Let the transfer function matrix $K(s)$ be SNI and the strictly proper transfer function matrix $P(s)$ be NI. Define $P_{2}=\lim _{s \rightarrow 0} s^{2} P(s), P_{1}=\lim _{s \rightarrow 0} s\left(P(s)-\frac{P_{2}}{s^{2}}\right)$, and $P_{0}=\lim _{s \rightarrow 0}\left(P(s)-\frac{P_{2}}{s^{2}}-\frac{P_{1}}{s}\right)$. Let $P_{2}=0$ and $P_{1} \neq 0$. Factorise $P_{1}=F_{1} V_{1}^{T}$ with $F_{1}$ and $V_{1}$ having full column rank such that $V_{1}^{T} V_{1}=I$. Suppose that $\mathbb{N}\left(P_{1}^{T}\right) \subseteq \mathbb{N}\left(P_{0}^{T}\right)$. Then the closed loop positive feedback interconnection between $P(s)$ and $K(s)$ is internally stable if and only if

$$
F_{1}^{T} K(0) F_{1}<0
$$

\section{B. Graph Theory}

Graphs are used to model information exchange among agents in a network. The information flow is bidirectional in an undirected graph whereas directional in a directed graph. We focus our attention here on directed graphs. A tutorial on graph theory can be found in [14]. A directed graph $\mathcal{G}=(\mathcal{V}, \mathcal{E})$ consists of a non-empty finite vertex set $\mathcal{V}=\left\{v_{1}, v_{2}, \ldots, v_{N}\right\}$ and an edge set $\mathcal{E} \subset \mathcal{V} \times \mathcal{V}$ of ordered pair of vertices called edges. An edge in $\mathcal{G}$ is denoted by $\left(v_{i}, v_{j}\right)$. If $\left(v_{i}, v_{j}\right) \in \mathcal{E}$, then agent $v_{j}$ can obtain information from agent $v_{i}$, but not necessarily vice versa. The set of neighbours of vertex $v_{i}$ is defined as $\mathcal{N}_{i}=\left\{v_{j} \in \mathcal{V}\right.$ : $\left.\left(v_{j}, v_{i}\right) \in \mathcal{E}\right\}$. Self edges are not allowed, that is, $\left(v_{i}, v_{i}\right) \notin \mathcal{E}$. A directed path in a graph from $v_{i}$ to $v_{j}$ is a sequence of edges of the form $\left(v_{i}, v_{i+1}\right),\left(v_{i+1}, v_{i+2}\right), \ldots,\left(v_{j-1}, v_{j}\right)$. A directed graph is strongly connected if there is a directed path from every vertex to every other vertex. The adjacency matrix $\mathcal{A}=\left[a_{i j}\right] \in \mathbb{R}^{N \times N}$ of $\mathcal{G}$ is defined as $a_{i j}=1$ if $\left(v_{j}, v_{i}\right) \in \mathcal{E}$, while $a_{i j}=0$ if $\left(v_{j}, v_{i}\right) \notin \mathcal{E}$. The Laplacian matrix $\mathcal{L}=\left[l_{i j}\right] \in \mathbb{R}^{N \times N}$ of $\mathcal{G}$ is defined as $l_{i i}=\sum_{j \neq i} a_{i j}$ and $l_{i j}=-a_{i j}$ for all $i \neq j$. A graph is called balanced if $\sum_{j=1}^{N} a_{i j}=\sum_{j=1}^{N} a_{j i}$ for all $i$.
Lemma 2 ([15], [16]): If $\mathcal{G}$ is strongly connected, then its Laplacian $\mathcal{L}$ satisfies:

1) $\operatorname{rank}(\mathcal{L})=N-1$

2) zero is a simple eigenvalue of $\mathcal{L}$ and $1_{N}$ is the corresponding eigenvector, i.e. $\mathcal{L} 1_{N}=0_{N}$ and the remaining $N-1$ eigenvalues all have positive real parts.

Lemma 3 ([15]): A directed graph $\mathcal{G}$ is balanced if and only if $1_{N}^{T} \mathcal{L}=0_{N}^{T}$.

Lemma 4 ([17]): Let $\mathcal{G}$ be a strongly connected and balanced graph. Then, $\mathcal{L}+\mathcal{L}^{T} \geq 0$ (i.e. positive semidefinite) with zero being its simple eigenvalue.

\section{PROBLEM Formulation}

Consider a group of $N$ homogeneous MIMO integrators with external disturbances acting on each system. The dynamics of the $i$ th system are described as

$$
y_{i}=w_{o_{i}}+P(s)\left(u_{i}+w_{i n_{i}}\right) \quad \forall i \in\{1, \ldots, N\},
$$

where $P(s)=\frac{k}{s} I_{m}$ is the transfer matrix with $k>0$, $u_{i}$ and $y_{i}$ are the control input and output of the $i$ th system respectively. Also, $w_{i n_{i}}$ and $w_{o_{i}}$ are input and output disturbances which are energy-bounded in an $\mathrm{H}_{2}$ (or in the time domain $\mathfrak{L}_{2}[0, \infty)$ ) sense. The information flow among the integrators is modelled by a directed graph $\mathcal{G}$ which is assumed balanced and strongly connected.

Following [8], the distributed control law is given by

$$
\begin{aligned}
& u_{i}=K(s) z_{i}, \\
& z_{i}=\sum_{j=1}^{N} a_{i j}\left(y_{i}-y_{j}\right),
\end{aligned}
$$

where $K(s)$ is an SNI feedback controller to each system, $z_{i}$ denotes the signal of relative output measurements and $a_{i j}$ are the elements of the adjacency matrix associated with the network graph $\mathcal{G}$. The collective network dynamics can be written as

$$
y=w_{o}+\left(I_{N} \otimes P(s)\right)\left(u+w_{i n}\right),
$$

and

$$
\begin{aligned}
& u=\left(I_{N} \otimes K(s)\right) z, \\
& z=\left(\mathcal{L} \otimes I_{m}\right) y,
\end{aligned}
$$

where $\mathcal{L}$ is the Laplacian matrix associated with the network graph $\mathcal{G}$. The closed loop networked system is shown in Fig. 1. By moving the block $\left(\mathcal{L} \otimes I_{m}\right)$ past the summing junction and letting $\bar{w}_{o}=\left(\mathcal{L} \otimes I_{m}\right) w_{o}$, we obtain the block diagram of Fig. 2. Note that $\bar{w}_{o}$ is a subset of $w_{o}$ since $\mathcal{L}$ is rank deficient. Consider the augmented plant $\bar{P}(s)$ to be the integration of the agents dynamics with the network topology; i.e. the transfer function from $u$ to $z$. Then $\bar{P}(s)$ can be written as $\bar{P}(s)=\left(\mathcal{L} \otimes I_{m}\right)\left(I_{N} \otimes P(s)\right)=(\mathcal{L} \otimes P(s))$. Also, let $\bar{K}(s)=\left(I_{N} \otimes K(s)\right)$ which is SNI since $K(s)$ is SNI.

Our objectives is first to show that $\bar{P}(s)$ is NI when $P(s)$ is a MIMO integrator and $\mathcal{L}$ corresponds to a directed, strongly connected and balanced graph. Then, we derive conditions 


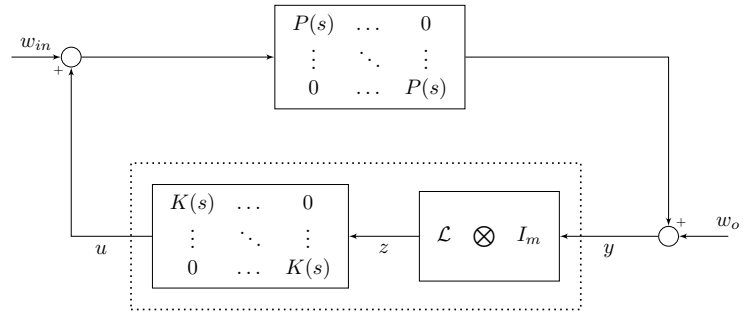

Fig. 1. Real physical networked system; general framework adopted from [8] but $\mathcal{L}$ here is for directed, strongly connected and balanced graphs.

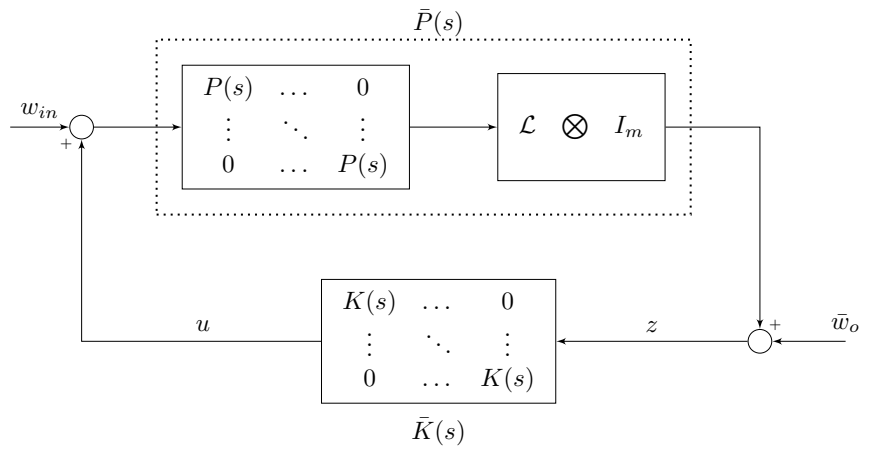

Fig. 2. Internal stability framework; general framework adopted from [8] but $\mathcal{L}$ here is for directed, strongly connected and balanced graphs.

using NI systems theory for which output consensus and cooperative tracking are achieved. This can be done since internal stability of the interconnection $[\bar{P}(s), \bar{K}(s)]$ in Fig. 2 is equivalent to consensus on the output $y$ in Fig. 1 by properties and the rank deficient of Laplacian matrix $\mathcal{L}$.

\section{MAIN RESULTS}

\section{A. Output consensus}

The following lemma states that the augmented plant $\bar{P}(s)$ is NI for a network of homogeneous MIMO integrators and directed information flow that is balanced and strongly connected.

Lemma 5: Consider a network of MIMO integrators with directed information flow $\mathcal{G}$ that is balanced and strongly connected. Then, $\bar{P}(s)$ is negative imaginary.

Proof: Since the network consists of MIMO integrators with $P(s)=\frac{k}{s} I_{m}$, it follows that $P(j \omega)^{*}=-P(j \omega)$. We need to show that $\bar{P}(s)$ is NI according to Definition 1. In fact we only need to show that $\bar{P}(s)$ satisfy conditions 1$)$, 2 ) and 4) since the agents are integrators. First, since $P(s)$ has a pole at the origin, then $\bar{P}(s)$ will have its poles at the origin as well. Consequently, condition 1) is satisfied. To show condition 2 ) is satisfied we have

$$
\begin{aligned}
j\left(\bar{P}(j \omega)-\bar{P}(j \omega)^{*}\right) & =j\left((\mathcal{L} \otimes P(j \omega))-\left(\mathcal{L}^{*} \otimes P(j \omega)^{*}\right)\right) \\
& =j\left((\mathcal{L} \otimes P(j \omega))-\left(\mathcal{L}^{*} \otimes-P(j \omega)\right)\right) \\
& =j\left((\mathcal{L} \otimes P(j \omega))+\left(\mathcal{L}^{*} \otimes+P(j \omega)\right)\right) \\
& =j\left(\left(\mathcal{L}+\mathcal{L}^{*}\right) \otimes P(j \omega)\right) \\
& =j\left(\left(\mathcal{L}+\mathcal{L}^{*}\right) \otimes \frac{k}{j \omega} I_{m}\right) \\
& =\frac{k}{\omega}\left(\left(\mathcal{L}+\mathcal{L}^{*}\right) \otimes I_{m}\right) \\
& =\frac{k}{\omega}\left(\left(\mathcal{L}+\mathcal{L}^{T}\right) \otimes I_{m}\right) \geq 0 \quad \forall \omega>0,
\end{aligned}
$$

by Lemma 4 since $\mathcal{G}$ is balanced and strongly connected, by properties of Kronecker product and by noting that $\mathcal{L}$ is real. Finally we have that $\lim _{s \rightarrow 0} s^{r} \bar{P}(s)=\lim _{s \rightarrow 0} s^{r}(\mathcal{L} \otimes$ $\left.\frac{k}{s} I_{m}\right)=0_{N m \times N m}$ for all $r \geq 3$ and $\lim _{s \rightarrow 0} s^{2} \bar{P}(s)=$ $\lim _{s \rightarrow 0} s^{2}\left(\mathcal{L} \otimes \frac{k}{s} I_{m}\right)=0_{N m \times N m}$.

Accordingly, $\bar{P}(s)$ is NI.

The following theorem gives a condition under which output consensus is achieved for a network of integrators with information flow that is balanced and strongly connected.

Theorem 1: Consider a network of MIMO integrators with directed information flow $\mathcal{G}$ that is balanced and strongly connected. Let $K(s)$ be a strictly negative imaginary feedback controller for each integrator. Then, output feedback consensus is achieved via control protocol (5) for networked system (4) as shown in Fig. 1 (or in a distributed manner (3) for each system (2)) under any external disturbances $w_{i n}, w_{o} \in \mathfrak{L}_{2}[0, \infty)$ if and only if $K(0)<0$.

Proof: Fist we need to prove that $[\bar{P}(s), \bar{K}(s)]$ as depicted in Fig. 2 is internally stable using Lemma 1. Then, internal stability of the interconnection $[\bar{P}(s), \bar{K}(s)]$ in Fig. 2 is equivalent to consensus on output $y$ in Fig. 1 by properties of Laplacian matrix $\mathcal{L}$ and due to $\left(\mathcal{L} \otimes I_{m}\right)$ being rank deficient. To this end, we have already shown in Lemma 5 that $\bar{P}(s)$ is NI. Also, $\bar{K}(s)=\left(I_{N} \otimes K(s)\right)$ is SNI since $K(s)$ is SNI. For $P(s)=\frac{k}{s} I_{m}$ we have $P_{2}=0_{m \times m}, P_{1}=k I_{m}$, and $P_{0}=0_{m \times m}$. This gives $\bar{P}_{2}=\left(\mathcal{L} \otimes P_{2}\right)=0_{N m \times N m}$, and $\bar{P}_{1}=\left(\mathcal{L} \otimes P_{1}\right)=\left(\mathcal{L} \otimes k I_{m}\right) \neq 0_{N m \times N m}$. Also, $\mathbb{N}\left(\bar{P}_{1}^{T}\right)=\mathbb{N}\left(\mathcal{L}^{T} \otimes P_{1}^{T}\right)=\left\{1_{N} \otimes b: b \in \mathbb{R}^{m \times 1}\right\} \cup\left\{0_{N m \times 1}\right\}$ and $\mathbb{N}\left(\bar{P}_{0}^{T}\right)=\mathbb{N}\left(\mathcal{L}^{T} \otimes P_{0}^{T}\right)=\left\{1_{N} \otimes b: b \in \mathbb{R}^{m \times 1}\right\} \cup$ $\left\{c \otimes d: c \in \mathbb{R}^{N \times 1}, d \in \mathbb{R}^{m \times 1}\right\}$ due to Lemma 3 and Lemma 2 in [8]. Consequently, $\mathbb{N}\left(\bar{P}_{1}^{T}\right) \subseteq \mathbb{N}\left(\bar{P}_{0}^{T}\right)$. As $\mathcal{G}$ is strongly connected we have $\operatorname{rank}(\mathcal{L})=N-1$. Thus, the singular value decomposition of $\mathcal{L}$ will have the form $\mathcal{L}=$ $\left[\begin{array}{ll}U_{1} & U_{2}\end{array}\right]\left[\begin{array}{cr}\Sigma & 0 \\ 0 & 0\end{array}\right]\left[\begin{array}{l}V_{1}^{T} \\ V_{2}^{T}\end{array}\right]=U_{1} \Sigma V_{1}^{T}$, with $U_{1} \in \mathbb{R}^{N \times N-1}$, $0<\Sigma \in \mathbb{R}^{N-1 \times N^{-1}}$, and $V_{1}^{T} \in \mathbb{R}^{N-1 \times N}$. Subsequently, $\bar{P}_{1}$ can be written as $\bar{P}_{1}=\left(\mathcal{L} \otimes k I_{m}\right)=\left(U_{1} \Sigma \otimes k\right)\left(V_{1} \otimes\right.$ $\left.I_{m}\right)^{T}=\bar{F}_{1} \bar{V}_{1}^{T}$.

Hence, $\bar{F}_{1}{ }^{T} \bar{K}(0) \bar{F}_{1}=\left(U_{1} \Sigma \otimes k\right)^{T}\left(I_{N} \otimes K(0)\right)\left(U_{1} \Sigma \otimes k\right)=$ $\left(\Sigma^{T} U_{1}^{T} U_{1} \Sigma \otimes k K(0) k\right)=\left(\Sigma^{T} \Sigma \otimes k^{2} K(0)\right)<0$ if and only if $K(0)<0$ since $\Sigma^{T} \Sigma>0$ with full rank of $N-1$ and $k>0$. We conclude that $[\bar{P}(s), \bar{K}(s)]$ is internally stable if and only if $K(0)<0$.

The proof that internal stability of $[\bar{P}(s), \bar{K}(s)]$ implies 
output consensus with and without external disturbances is similar to that of Theorem 1 in [8] with the only difference being that here $\mathcal{L} 1_{N}=0_{N}$ holds because $\mathcal{G}$ is strongly connected.

\section{B. Cooperative tracking to a pre-defined fixed reference}

In this section we show that the NI stability theory can be used to solve a cooperative tracking problem for multiple integrator NI systems. The distributed control law now becomes

$$
\begin{aligned}
& u_{i}=K(s) z_{i}, \\
& z_{i}=\sum_{j=1}^{N} a_{i j}\left(y_{i}-y_{j}\right)+d_{i}\left(y_{i}-r\right),
\end{aligned}
$$

for all $i \in\{1, \ldots, N\}$ where $r$ is the fixed reference and $d_{i}=1$ if agent $i$ is connected to the reference and $d_{i}=0$ otherwise. The collective dynamics of the control law can be written as

$$
\begin{aligned}
& u=\left(I_{N} \otimes K(s)\right) z, \\
& z=\left((\mathcal{L}+D) \otimes I_{m}\right)\left(y-1_{N} r\right) .
\end{aligned}
$$

The plant from $u$ to $z$ is $\bar{P}(s)=\left((\mathcal{L}+D) \otimes I_{m}\right)\left(I_{N} \otimes\right.$ $P(s))=((\mathcal{L}+\mathcal{D}) \otimes P(s))$ which is NI following similar steps as in Lemma 5 and noting that $(\mathcal{L}+\mathcal{D})+(\mathcal{L}+\mathcal{D})^{T}$ is positive definite since $\mathcal{D}$ is nonzero diagonal matrix, and by Lemmas 2 and 4 .

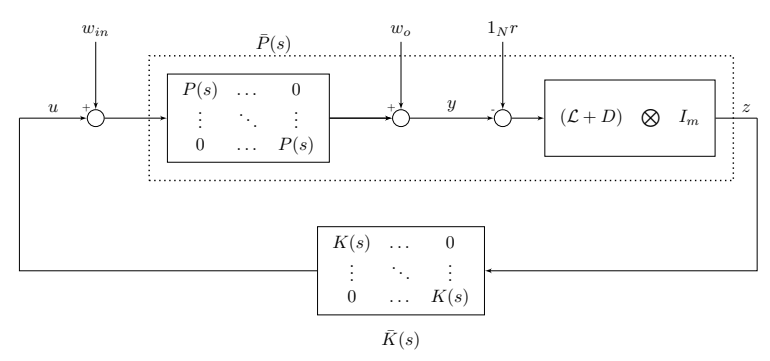

Fig. 3. Cooperative output tracking block diagram.

The following theorem gives a condition under which cooperative output tracking to a fixed reference is achieved for a network of MIMO integrators with directed information flow that is balanced and strongly connected.

Theorem 2: Consider a network of MIMO integrators with directed information flow $\mathcal{G}$ that is balanced and strongly connected. Let $K(s)$ be a strictly negative imaginary feedback controller for each integrator. Then, cooperative output tracking to a fixed reference $r$ is achieved via control protocol (7) for networked system (4) as shown in Fig. 3 (or in a distributed manner (6) for each system (2)) under any external disturbances $w_{i n}, w_{o} \in \mathfrak{L}_{2}[0, \infty)$ if and only if $K(0)<0$.

Proof: Following similar steps as in the proof of Theorem 1, $[\bar{P}(s), \bar{K}(s)]$ is internally stable if and only if $K(0)<0$. Subsequently, internal stability of $[\bar{P}(s), \bar{K}(s)]$ yields $z \rightarrow 0$ in (7). That is, $z_{i} \rightarrow 0 \forall i \in\{1, \ldots, N\}$ in (6). Hence, $y_{i} \rightarrow y_{j} \forall j \in \mathcal{N}_{i}, j \neq i$ with $d_{i}=0$ and $y_{i} \rightarrow r$ when $d_{i}=1$. Consequently, cooperative output tracking to a fixed reference $r$ is achieved, i.e., $y_{i}=y_{j}=r$ $\forall i \in\{1, \ldots, N\}$ and $\forall j \in \mathcal{N}_{i}, j \neq i$.

Remark 1: A simple DC gain condition, that can easily be satisfied, guarantees output consensus and tracking via the distributed control law proposed in this paper. This can be considered an advantage over other existing protocols in literature, for example [18] where state space techniques and finding suitable gain matrices to satisfy certain conditions are required in the design.

\section{Application}

In this section we apply the theoretical results of Section IV-B to achieve rendezvous of nonholonomic WMR.

\section{A. Robot model}

Consider a group of $N=3$ homogeneous WMR. The kinematic model of the $i$ th robot is given by

$$
\begin{aligned}
\dot{x}_{i} & =v_{i} \cos \phi_{i}, \\
\dot{y}_{i} & =v_{i} \sin \phi_{i}, \quad \forall i \in\{1,2,3\} \\
\dot{\phi}_{i} & =w_{i} .
\end{aligned}
$$

where $\left(x_{i}, y_{i}\right)$ is the position of the $i$ th robot and $\phi_{i}$ is its orientation. Also, $v_{i}$ and $\omega_{i}$ are the linear and angular velocities of the $i$ th robot respectively. Although we limit the work here to three robots, more robots can be considered without complexity. In this paper, we are interested in the rendezvous problem defined as follows:

Problem statement 1: Find a distributed control law $v_{i}$ and $w_{i}$ for each WMR such that all WMR reach a pre-defined fixed rendezvous position $\left(x_{r}, y_{r}\right)$ which is available only to some of the WMR.

In order to apply NI cooperative tracking results of Section IV-B to the WMR, we apply input-output linearisation such that the system between the new input and output is linear (see e.g. [19], [20] for more information). Define two new outputs to be controlled as

$$
\begin{aligned}
\tilde{x}_{i} & =x_{i}+l \cos \phi_{i} \\
\tilde{y}_{i} & =y_{i}+l \sin \phi_{i}
\end{aligned}, \quad \forall i \in\{1,2,3\}
$$

where $l \neq 0$ is the distance from $\left(\tilde{x}_{i}, \tilde{y}_{i}\right)$ to $\left(x_{i}, y_{i}\right)$. Using the kinematic model (8), the new dynamics of the WMR is given by

$$
\left[\begin{array}{c}
\dot{\tilde{x}}_{i} \\
\dot{\tilde{y}}_{i}
\end{array}\right]=F\left(\phi_{i}\right)\left[\begin{array}{c}
v_{i} \\
\omega_{i}
\end{array}\right]
$$

where $F\left(\phi_{i}\right)=\left[\begin{array}{cc}\cos \phi_{i} & -l \sin \phi_{i} \\ \sin \phi_{i} & l \cos \phi_{i}\end{array}\right]$. Define new inputs to be $u_{1 i}, u_{2 i}$. Using these two new control variables we get

$$
\left[\begin{array}{c}
v_{i} \\
\omega_{i}
\end{array}\right]=F\left(\phi_{i}\right)^{-1}\left[\begin{array}{l}
u_{1 i} \\
u_{2 i}
\end{array}\right]
$$

and the robot's linearised model becomes

$$
\begin{aligned}
& \dot{\tilde{x}}_{i}=u_{1 i} \\
& \dot{\tilde{y}}_{i}=u_{2 i} .
\end{aligned}, \quad \forall i \in\{1,2,3\}
$$


The dynamic of system (12) is linear and decoupled and corresponds to an integrator NI system; i.e. $P(s)=\frac{1}{s} I_{2}$ which implies that the algorithms of the previous section can be directly applied.

\section{B. Simulation results}

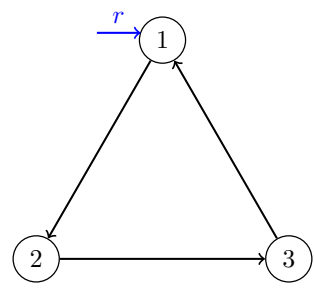

Fig. 4. Network graph.

The initial positions (in metres) and orientation (in degrees) are chosen as $(0.7,0.4,0),(0.3,0.4,0)$ and $(0.5,0.1,0)$ for the three robots. Also, we assume in this simulation that $l=0.03 \mathrm{~m}$. The final rendezvous position (in metres) is $(0.5,0.3)$. Note that with this approach the orientation is left uncontrolled. The network graph that models the communication links among the three robots is shown in Fig. 4. Also, the reference is available only to system 1 . Hence, the Laplacian matrix associated with $\mathcal{G}$ and the diagonal matrix $\mathcal{D}$ are

$$
\mathcal{L}=\left[\begin{array}{ccc}
1 & 0 & -1 \\
-1 & 1 & 0 \\
0 & -1 & 1
\end{array}\right], \quad \mathcal{D}=\left[\begin{array}{lll}
1 & 0 & 0 \\
0 & 0 & 0 \\
0 & 0 & 0
\end{array}\right]
$$

An SNI controller for each robot is chosen as $K(s)=$ $\frac{-3 s-2}{s+1} I_{2}$ with zero initial condition which satisfies $K(0)=$ $-2 I_{2}<0$. Consequently, rendezvous is achieved via the cooperative output position tracking results of Theorem 2 as shown in Fig. 5 without and with external input disturbances.

\section{Experimental results}

To validate the proposed control mechanism using realrobot experiments, miniature mobile robots, Mona [21] which is a low-cost and open-source mobile robot platform, were deployed. Mona has been developed for use in the research of swarm robotics [22]. Experiments were conducted in a rectangular arena with dimensions of $1.4 \mathrm{~m} \times 0.9 \mathrm{~m}$ which has been developed for study on long-term swarm robotics scenarios [23]. An open-source multi-robot tracking system [24] which tracks both the position and orientation of the robot using an overhead camera was used. Via a unique circular tag attached on the surface of each robot, the position and orientation can be tracked. The position information is sent to the controller via a ROS communication framework. Three Mona robots where used in the experiment. The initial positions of the three robots were $(0.6942,0.376) \mathrm{m}$, $(0.3296,0.4415) \mathrm{m}$, and $(0.4409,0.1194) \mathrm{m}$. The final rendezvous position was $(0.5,0.3) \mathrm{m}$. Also, $l=0.03 \mathrm{~m}$ for the robots. The same network graph of Fig. 4 was used. The controller to each Mona robot was the same as the one

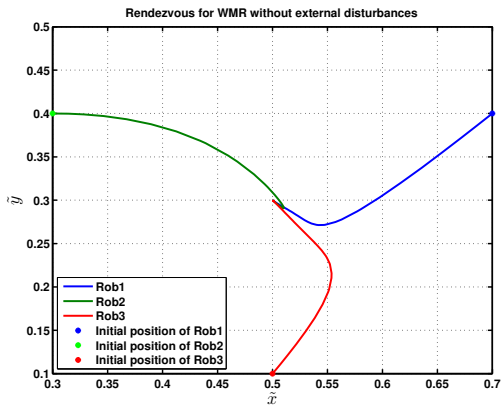

(a)

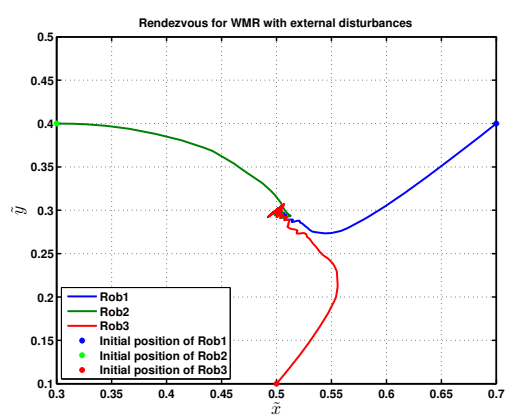

(b)

Fig. 5. Rendezvous for WMR, (a) without disturbances and (b) with disturbances.

designed in Section V-B. Snapshots of the positions of the three Mona robots during the experiment at different time durations is shown in Fig. 6. As can be seen from the figure, the Mona robots rendezvous at the desired reference position. The position trajectory of the three Mona robots is shown in Fig. 7. Note that in order to avoid collision during the experiment, each robot stops when it is near the rendezvous point $(0.05 \mathrm{~m}$ range $)$. Finally, the control inputs are shown in Fig. 8.

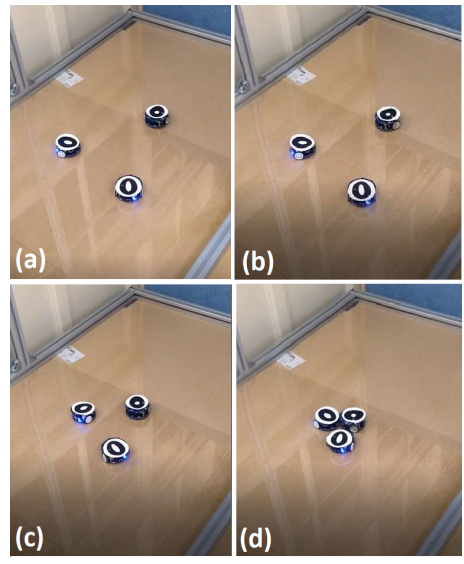

Fig. 6. Position snapshots of the 3 Mona robots at different time durations starting from initial position and ending in rendezvous. (a) $t=0 \mathrm{~s}$. (b) $t=2 \mathrm{~s}$. (c) $t=5 \mathrm{~s}$. (d) $t=11 \mathrm{~s}$.

\section{CONCLUSION}

A rendezvous problem for multiple WMR was tackled via NI cooperative tracking results proposed in this paper. 


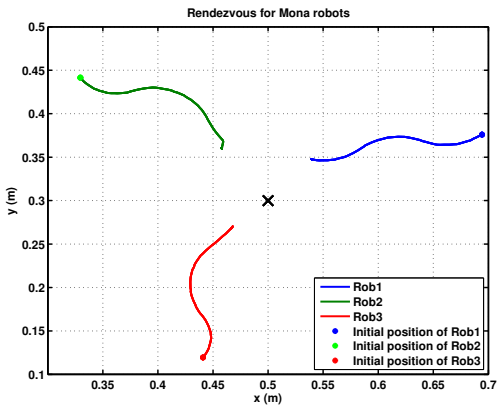

Fig. 7. Position trajectory and rendezvous of three Mona robots to final position $(0.5,0.3) \mathrm{m}$ marked in cross.

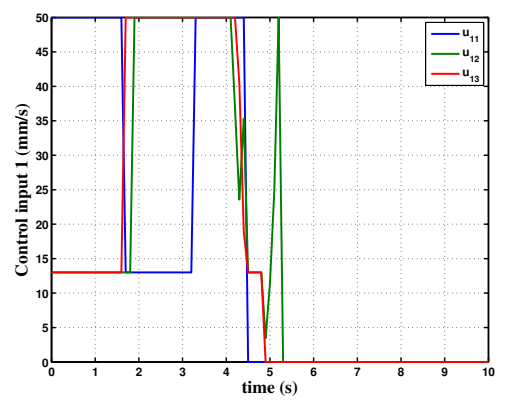

(a)

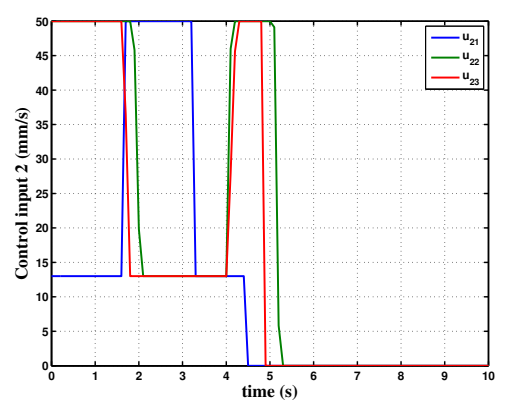

(b)

Fig. 8. The control input which represents the desired (reference) velocity to each motor. (a) Control input 1. (b) Control input 2.

Experimental results, using Mona robots, as well as simulation results showed that rendezvous is achieved via the distributed control protocol proposed with communication among the robots being modelled by directed graphs that are balanced and strongly connected. Extending the current work to formation control such as [25], [26] can be considered in future research.

\section{REFERENCES}

[1] G. Antonelli, F. Arrichiello, F. Caccavale, and A. Marino, "Decentralized time-varying formation control for multi-robot systems," International Journal of Robotics Research, vol. 33, no. 7, pp. 10291043, 2014.

[2] W. Dong, "Tracking control of multiple-wheeled mobile robots with limited information of a desired trajectory," IEEE Transactions on Robotics, vol. 28, no. 1, pp. 262-268, 2012.

[3] W. Ren, H. Chao, W. Bourgeous, N. Sorensen, and Y. Chen, "Experimental validation of consensus algorithms for multivehicle cooperative control," IEEE Transactions on Control Systems Technology, vol. 16, no. 4, pp. 745-752, 2008.
[4] A. Lanzon and I. R. Petersen, "Stability robustness of a feedback interconnection of systems with negative imaginary frequency response," IEEE Transactions on Automatic Control, vol. 53, no. 4, pp. 10421046, May 2008.

[5] H. J. Chen and A. Lanzon, "Closed-loop stability analysis of discretetime negative imaginary systems," Systems \& Control Letters, vol. 114, pp. 52-58, Apr. 2018.

[6] A. Lanzon and H. J. Chen, "Feedback stability of negative imaginary systems," IEEE Transactions on Automatic Control, vol. 62, no. 11, pp. 5620-5633, Nov. 2017.

[7] I. R. Petersen, "Negative imaginary systems theory and applications," Annual Reviews in Control, vol. 42, pp. 309-318, 2016.

[8] J. Wang, A. Lanzon, and I. R. Petersen, "Robust output feedback consensus for networked negative-imaginary systems," IEEE Transactions on Automatic Control, vol. 60, no. 9, pp. 2547-2552, Sep. 2015.

[9] _ - "Robust cooperative control of multiple heterogeneous negativeimaginary systems," Automatica, vol. 61, pp. 64-72, Nov. 2015.

[10] O. Skeik and A. Lanzon, "Robust stabilization of networked multiagent systems with strict negative imaginary uncertainties: An LMI approach," in Proceedings of the 16th European Control Conference, Limassol, Cyprus, Jun. 2018, pp. 167-171.

[11] — - "Robust output consensus for networks of homogeneous negative imaginary systems," in Proceedings of the 57th IEEE Conference on Decision and Control, Miami, FL, USA, Dec. 2018, pp. 3836-3841.

[12] M. A. Mabrok, A. G. Kallapur, I. R. Petersen, and A. Lanzon, "Generalizing negative imaginary systems theory to include free body dynamics: Control of highly resonant structures with free body motion," IEEE Transactions on Automatic Control, vol. 59, no. 10, pp. 2692-2707, Oct. 2014.

[13] J. Xiong, I. R. Petersen, and A. Lanzon, "A negative imaginary lemma and the stability of interconnections of linear negative imaginary systems," IEEE Transactions on Automatic Control, vol. 55, no. 10, pp. 2342-2347, Oct. 2010.

[14] W. Ren, R. Beard, and E. Atkins, "Information consensus in multivehicle cooperative control," IEEE Control Systems Magazine, vol. 27, no. 2, 2007.

[15] R. Olfati-Saber and R. Murray, "Consensus problems in networks of agents with switching topology and time-delays," IEEE Transactions on Automatic Control, vol. 49, no. 9, pp. 1520-1533, Sep. 2004.

[16] P. Lin and Y. Jia, "Average consensus in networks of multi-agents with both switching topology and coupling time-delay," Physica A: Statistical Mechanics and its Applications, vol. 387, no. 1, pp. 303313, 2008.

[17] W. Liu, S. Zhou, Y. Qi, and X. Wu, "Leaderless consensus of multi-agent systems with Lipschitz nonlinear dynamics and switching topologies," Neurocomputing, vol. 173, pp. 1322-1329, 2016.

[18] X. Ding, "On dynamic distributed control and its application to multirobot systems," in Chinese Control Conference, CCC, 2017, pp. 88638868.

[19] S. G. Tzafestas, Introduction to Mobile Robot Control. Elsevier, 2014.

[20] A. De Luca, G. Oriolo, and M. Vendittelli, Control of Wheeled Mobile Robots: An Experimental Overview. Berlin, Heidelberg: Springer Berlin Heidelberg, 2001, pp. 181-226.

[21] F. Arvin, J. Espinosa, B. Bird, A. West, S. Watson, and B. Lennox, "Mona : an Affordable Open-Source Mobile Robot for Education and Research," Journal of Intelligent \& Robotic Systems, May 2018.

[22] F. Arvin, S. Watson, A. Emre Turgut, J. Espinosa, T. Krajnik, and B. Lennox, "Perpetual robot swarm: Long-term autonomy of mobile robots using on-the-fly inductive charging," Journal of Intelligent \& Robotic Systems, Oct. 2017.

[23] F. Arvin, T. Krajník, and A. E. Turgut, "P $\Phi S S$ : An Open-source Experimental Setup for Real-world Implementation of Swarm Robotic Systems in Long-term Scenarios," in 5th International Conference Modelling and Simulation for Autonomous, Oct. 2018.

[24] T. Krajník, M. Nitsche, J. Faigl, P. Vaněk, M. Saska, L. Přeučil, T. Duckett, and M. Mejail, "A practical multirobot localization system," Journal of Intelligent \& Robotic Systems, vol. 76, no. 3, pp. 539-562, Dec 2014.

[25] J. Hu, P. Bhowmick, and A. Lanzon, "Distributed adaptive timevarying group formation tracking for multi-agent systems with multiple leaders on directed graphs," IEEE Transactions on Control of Network Systems, In press, DOI:10.1109/TCNS.2019.2913619.

[26] J. Hu and A. Lanzon, "An innovative tri-rotor drone and associated distributed aerial drone swarm control," Robotics and Autonomous Systems, vol. 103, pp. 162-174, 2018. 